\title{
Techniques for obtaining differential cell counts from bone marrow trephine biopsy specimens
}

\author{
BRIDGET S WILKINS, C J O'BRIEN From the University Department of Pathology, Leeds General \\ Infirmary
}

SUMMARY Differential cell counts were performed on 200 paired bone marrow aspirates and trephine biopsy specimens to compare the distribution of cell types. Relatively more immature myeloid cells were found in the trephine biopsy specimens and relatively more polymorphs and lymphocytes in the aspirates. Two methods for sampling areas of the trephine biopsy specimens for counting were assessed, and the differences between aspirates and trephine specimens were found to be more consistent when the second, more extensive, sampling method was used. This method also permitted quantiation of some features of bone marrow topography and provided information that would not normally be obtainable from aspirated material.

The techniques were easy to apply and took relatively little time to perform. They could offer useful information in the study of bone marrow disorders, particularly those such as myelodysplastic syndromes in which disturbances of marrow architecture are prominent.

In the diagnosis and study of diseases of the bone marrow, samples obtained by aspiration and by trephine biopsy can provide complementary information. ${ }^{12}$ Both types of sample can be assessed in terms of cytological appearances, and trephine biopsy specimens can provide additional information about marrow architecture.

Quantitative assessment of marrow aspirates is made in many centres by performing differential cell counts. It is not usual for similar quantitative assessment of marrow trephine biopsy specimens to be attempted, although there are theoretical advantages in doing so. Aspiration may result in dilution of the marrow by peripheral blood ${ }^{3-5}$ and may preferentially sample less adherent marrow cells. Trephining should avoid these problems, and cell counts from marrow trephines could also provide a means of quantifying changes in the spatial arrangement of haemopoietic cells seen in a variety of disease states.

We used two methods to perform differential cell counts in bone marrow trephine biopsy specimens and we compared the results with counts obtained from aspirates. The effect of peripheral blood dilution on the aspirates was highlighted, and there was some evidence to support the hypothesis that aspiration techniques sample the most immature haemopoietic cells incompletely. One of our cell counting techniques also enabled us to show quantitatively features of bone marrow topography.

Accepted for publication 30 December 1987

\section{Material and methods}

Two hundred paired bone marrow aspirates and trephine biopsy specimens, received as part of the routine workload in the pathology department at Leeds General Infirmary were studied. The only criteria for selection were that both samples should have been obtained from each patient on the same occasion and that both should be technically satisfactory in terms of quantity of marrow and quality of cytological detail. A wide range of diagnoses was encompassed.

The aspirated material was examined as air dried, Giemsa stained smears. The trephine biopsy specimens were fixed in $10 \%$ formalin and embedded in plastic ("Immuno-Bed", Polysciences Inc, Pennsylvania, USA) without decalcification. ${ }^{6}$ Sections $2 \mu \mathrm{m}$ thick, stained with haematoxylin and eosin, were examined.

The aspirates were sampled by counting 100 cells in the trail immediately behind each of three particles (fig 1a). The two trephine sampling methods are also shown in fig 1. By the first method (fig 1b), 100 cells were counted in each of three sites (paratrabecular, central, and intermediate) using a different marrow space for each site.

In the second method (fig 1c) a grid square graticule (Graticules Limited, Tonbridge, England) was used in the microscope eyepiece to define the area of cells to be counted, and the differential count was performed on a strip of cells $0.15 \mathrm{~mm}$ wide across the full width of the marrow space. The strip was divided into zones, numbered 1 to 5 , depending on position relative to the 


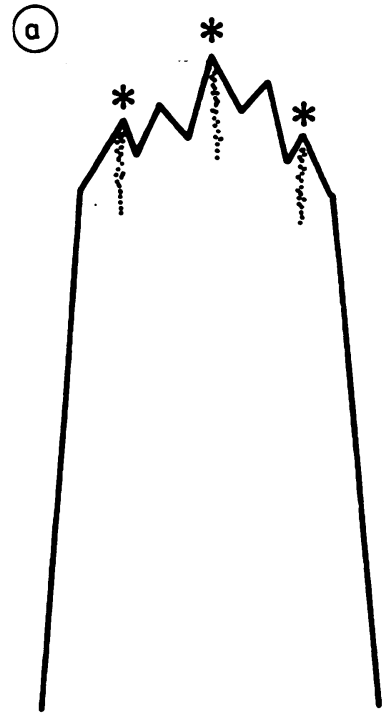

* Bone marrow particle
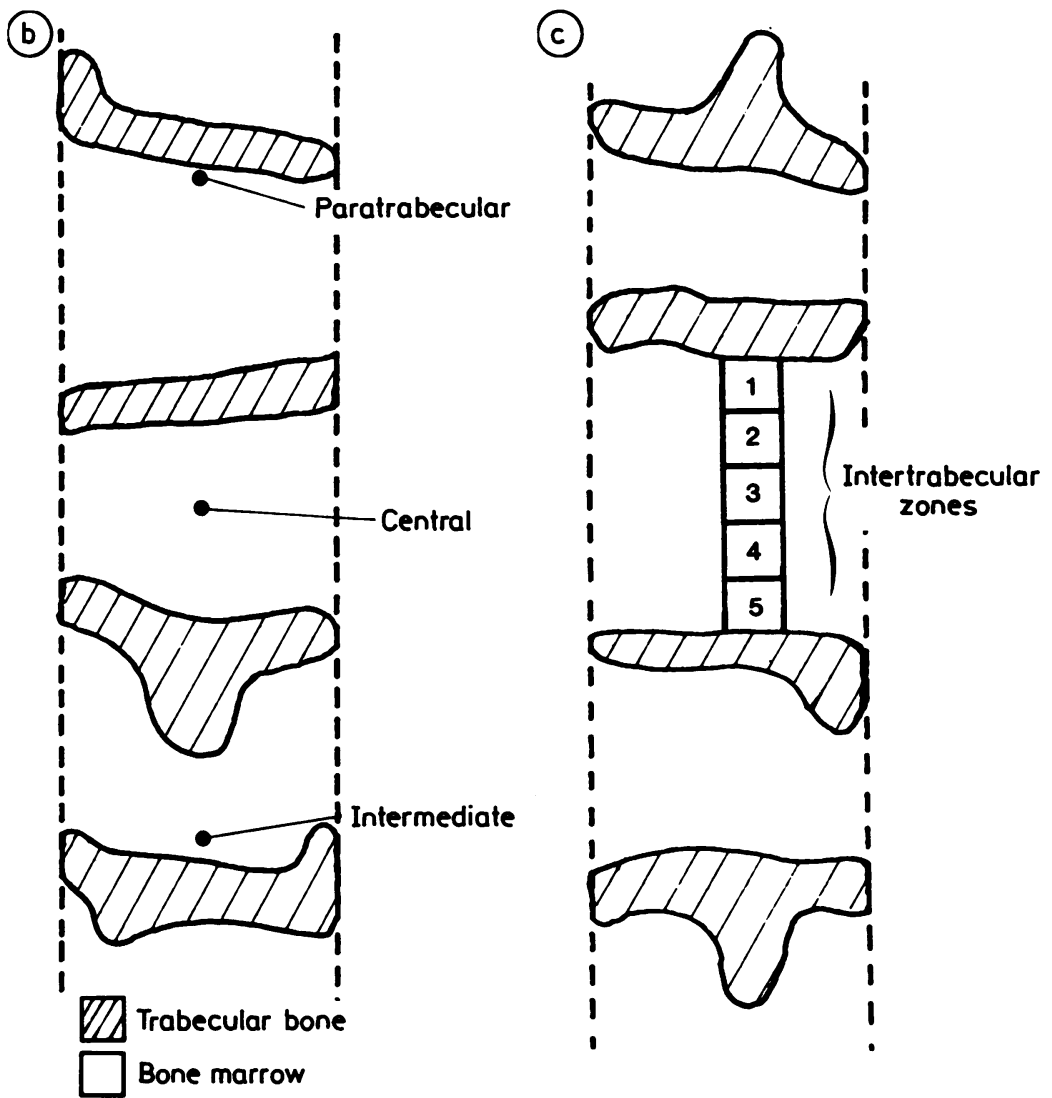

Fig 1 Sampling methods used for performing differential cell counts in bone marrow aspirates and trephine biopsy specimens.

limiting trabeculae of the marrow space, so that it was possible to compare cell counts obtained from the different zones. Differential cell counts were performed using this sampling method in two marrow spaces in each trephine biopsy specimen. The spaces were

Table Comparison of aspirate and trephine differential cell counts using the second trephine sampling method

\begin{tabular}{llrl}
\hline Cell type & $M(A-T)^{*}$ & $\mathrm{P}$ & $\mathrm{r}$ \\
\hline Monocytes & +1.004 & 0.001 & -0.022 \\
Plasma cells & +0.070 & 0.747 & +0.324 \\
Blasts & +1.373 & 0.534 & +0.909 \\
Promyelocytes & -4.611 & $<0.001$ & +0.549 \\
Myelocytes & -6.086 & $<0.001$ & +0.711 \\
Metamyelocytes & -0.578 & 0.552 & +0.725 \\
Polymorphs & +7.390 & $<0.001$ & +0.871 \\
Lymphoid cells & +5.641 & 0.001 & +0.952 \\
Erythroid cells & -4.967 & 0.007 & +0.739
\end{tabular}

*Mean difference between aspirate and trephine differential cell counts $(\%) ; p=$ probability of difference between counts arising by chance; $r=$ product-moment coefficient of correlation; (perfect correlation $=+1$ or -1 ; absence of correlation $=0$ ). selected to be of roughly average size $(0.74 \mathrm{~mm}$ to 1.48 $\mathrm{mm}$ in width between trabeculae), bounded by parallel trabeculae of bone, and separated by at least one other marrow space. Thirty trephines from the original 200 were sampled by this second method.

In all cases counts were performed without prior knowledge of the diagnosis.

A manual laboratory counter (Clay Adams, Parsipanny, New Jersey, USA), of a type that is routinely used in haematological practice, was used to count nine cell types (table). A tenth group of miscellaneous cells was also counted including megakaryocytes, histiocytes, spindle cells and metastatic carcinoma cells, but their numbers were too small for statistical analysis.

For each aspirate or trephine the average count of each cell type was calculated and converted into a percentage. Comparisons between cell counts were made using the paired Student's $t$ test, having first shown that their frequency distributions formed normal curves with only slight or moderate skew. Correla- 


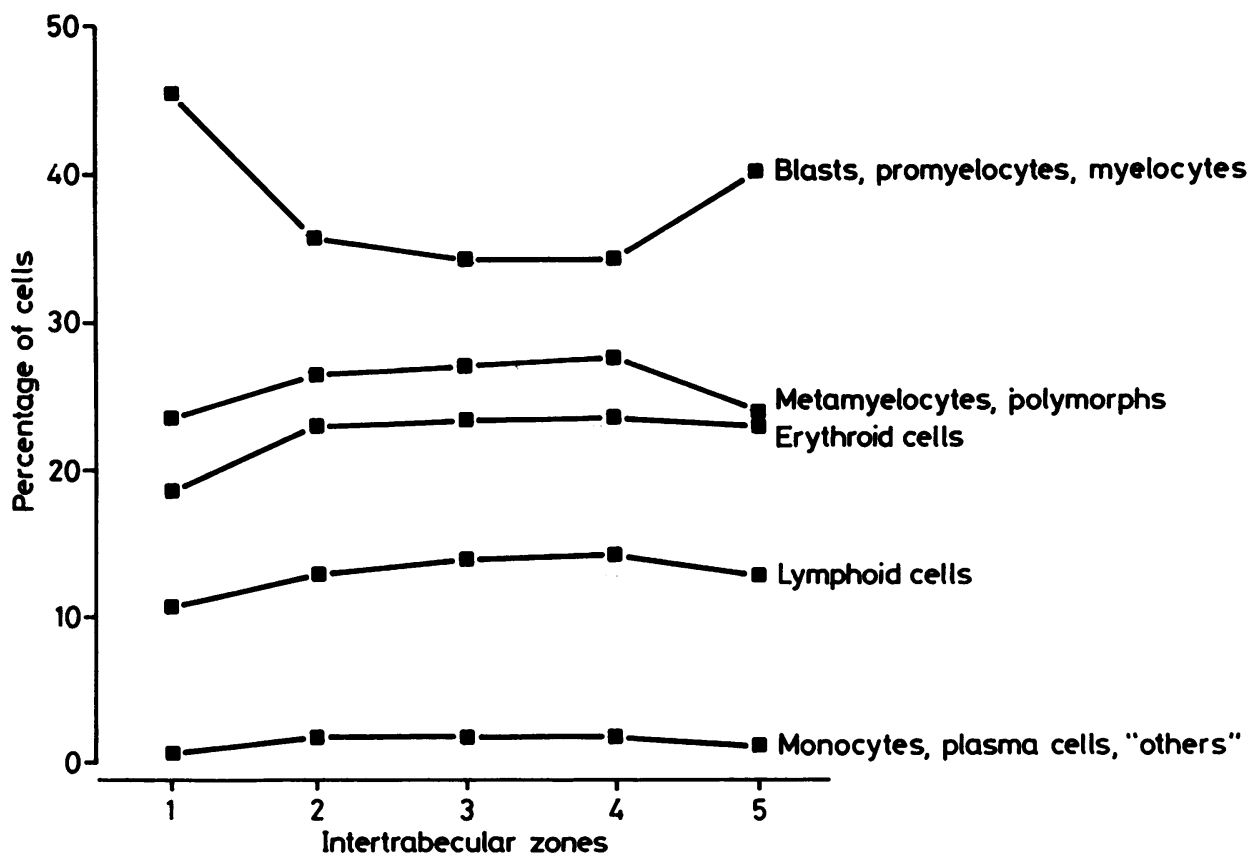

Fig 2 Distribution of cell types within marrow spaces as shown by performing differential cell counts in zones across spaces.

tions were measured by calculation of the productmoment correlation coefficient.

\section{Results}

The table shows the results obtained when aspirate and trephine differential cell counts were compared, using the second trephine sampling method. The differences were significant $(p<0.05)$ for cell types other than plasma cells, blasts, and metamyelocytes. The degree of correlation ( $r$ ) between aspirate and trephine cell counts was high. A similar pattern of results was seen when counts obtained by the first trephine sampling method were compared with the aspirate counts, but the correlations were weaker, and we considered that less accurate sampling of the trephine biopsy marrow cells had been achieved.

Recognition of the different cell types in trephine biopsy specimens was generally easy, although monocytes and their precursors were often difficult to identify. The true percentages of these cells are probably higher than those we achieved. Only a small number of cells were unidentifiable and these were not included in the cell counts. Occasional unidentifiable cells and larger numbers of bare nuclei were also found in the aspirates, and these too were excluded from the counts.

The differential cell counts within individual zones across the full width of intertrabecular spaces are shown in fig 2 . The patterns of early and late myeloid cell distribution are clear, and, although less pronounced, the tendency for erythroid cells to lie centrally within marrow spaces can be seen. For promyelocytes, myelocytes, polymorphs and erythroid cells, the differences in percentage cell counts between one or both of the paratrabecular zones (zones 1 and 5) and the central zone (zone 3$)$ were significant $(p<0.05)$.

\section{Discussion}

We have attempted to devise methods by which differential cell counting can be performed on bone marrow trephine biopsy specimens to obtain information comparable with or complementary to that gained from similar counts carried out on aspirated bone marrow.

Performing differential cell counts on trephines by the first sampling method was rapid (five minutes for each case) and the technique could be applied to most of the trephine biopsy specimens received routinely in the department. The second method was more time consuming (15 to 20 minutes for each case), but seemed to provide a more representative sample, as judged by the closer correlations between aspirate and trephine biopsy cell counts. Many fewer trephine biopsy specimens could be assessed by this method, however, because of the strict morphological criteria which we imposed to standardise the sampling. 
The results show that with our sampling and counting methods the trephine biopsy specimens contained a relative excess of immature myeloid cells and the aspirates a relative excess of potymorphs and lymphocytes. The greater numbers of polymorphs and lymphocytes counted in the aspirates are thought to be due mainly to dilution of the marrow by peripheral blood, and possibly also to preferential aspiration of more mature cells. Peripheral blood dilution of bone marrow aspirates has been shown in a variety of contexts. ${ }^{3-5}$ Holdrinet et al found that peripheral blood cells accounted for $14 \pm 8 \%$ of the nucleated cells counted in marrow aspirates from healthy volunteers, ${ }^{5}$ emphasising how variable the contamination can be.

The second sampling method provided a quantitative representation of the architectural organisation of marrow within intertrabecular spaces (fig 2), as described by Burkhardt et al. ${ }^{78}$ Early myeloid precursors tend to lie close to the trabeculae, with granulocyte maturation occurring progressively towards the centres of the marrow spaces. Erythroid cell clusters and megakaryocytes tend to be associated with central sinusoids. Cells in peripheral sites may be less readily aspirated than those in central areas, and the immature myeloid cells may possibly be more adherent to the marrow stroma than more mature forms. The fact that the percentage counts of blasts did not differ significantly between aspirates and trephine biopsy specimens seems to contradict these hypotheses. In the total sariple of cases, however, the blasts were a heterogeneous population, not all of myeloid lineage, and when cases showing no abnormality were studied separately (data not shown) the difference did reach significance.

The proportions of cell types in aspirated marrow therefore may not accurately reflect those present in marrow that has not been disrupted or diluted. Obtaining marrow by trephine biopsy should avoid these sources of error, and our results show that differential cell counts from these specimens can provide information complementary to that available from aspirates.

In this study we did not attempt to apply our cell counting techniques to the analysis of particular bone marrow pathologies. We envisage, however, that they will be of use in the study of diseases such as reactive and neoplastic myeloproliferative states and the myelodysplastic syndromes, in which disturbances of marrow architecture are prominent. Analysis of the abnormal localisation of immature myeloid precursors and erythroid nests in myelodysplasia described by Tricot $e t$ al, ${ }^{9}$ may be assisted by such cell counting techniques.

Differential cell counts from marrow trephine biopsy specimens may also be found useful in diagnostic practice in cases in which aspiration yields a "dry tap," containing only peripheral blood cells.

\section{References}

1 Block M. Bone marrow examination. Arch Pathol Lab Med 1976;100:454-6.

2 Pasquale D, Chikkappa G. Comparative evaluation of bone marrow aspirate particle smears, biopsy imprints and biopsy sections. Am J Haematol 1986;22:381-9.

3 Dresch C, Faille A, Poirier O, Kadouche J. The cellular composition of the granulocyte series in the normal human bone marrow according to the volume of the sample. J Clin Pathol 1974;27:106-8.

4 Gordon M, Douglas I. The effect of peripheral blood contamination on colony yield from human bone marrow aspirates. Exp Haematol 1977;5:274-80.

5 Holdrinet R, von Egmond J, Wessels J, Haanen C. A method for quantification of peripheral blood admixture in bone marrow aspirates. Exp Haematol 1980;8:103-7.

6 Islam A, Frisch B. Plastic embedding in routine histology 1: Preparation of semi-thin sections of undecalcified marrow cores. Histopathology 1985;9:1263-74.

7 Frisch B, Lewis S, Burkhardt R, Bartl R. Biopsy pathology of bone and bone marrow. London: Chapman and Hall, 1985;28-43.

8 Bartl R, Frisch B, Burkhardt R. Bone marrow biopsies revisited. 2nd Edition. Basel: Karger, 1985:9-15.

9 Tricot G, de Wolf-Peeters C, Hendrickx B, Verwilghen R. Histological findings in myelodysplastic syndromes and comparison with bone marrow smears. Br J Haematol 1984;57:42330.

Requests for reprints to: Dr Bridget S Wilkins, Department of Pathology, Southampton General Hospital, Tremona Road, Southampton, SO9 4XY, England. 